\title{
Nanofiber mat spinal cord dressing-released glutamate impairs blood-spinal cord barrier
}

\author{
Dorota Sulejczak ${ }^{1}$, Anna Taraszewska², Stanisław J. Chrapusta ${ }^{1}$, Dorota Dziewulska ${ }^{2,3}$, Paweł Nakielski ${ }^{4}$, \\ Janina Rafałowska² \\ ${ }^{1}$ Department of Experimental Pharmacology, Mossakowski Medical Research Centre, Polish Academy of Sciences, Warsaw, \\ 2Department of Experimental and Clinical Neuropathology, Mossakowski Medical Research Centre, Polish Academy of Sciences, \\ Warsaw, ${ }^{3}$ Department of Neurology, Medical University of Warsaw, Warsaw, ${ }^{4}$ Department of Mechanics and Physics of Fluids, \\ Institute of Fundamental Technological Research, Polish Academy of Sciences, Warsaw, Poland
}

\begin{abstract}
An excessive glutamate level can result in excitotoxic damage and death of central nervous system (CNS) cells, and is involved in the pathogenesis of many CNS diseases. It may also be related to a failure of the blood-spinal cord barrier (BSCB). This study was aimed at examining the effects of extended administration of monosodium glutamate on the BSCB and spinal cord cells in adult male Wistar rats. The glutamate was delivered by subarachnoidal application of glutamate-carrying electrospun nanofiber mat dressing at the lumbar enlargement level. Half of the rats with the glutamate-loaded mat application were treated systemically with the histone deacetylase inhibitor valproic acid. A group of intact rats and a rat group with subarachnoidal application of an 'empty' (i.e., carrying no glutamate) nanofiber mat dressing served as controls. All the rats were euthanized three weeks later and lumbar fragments of their spinal cords were harvested for histological, immunohistochemical and ultrastructural studies. The samples from controls revealed normal parenchyma and BSCB morphology, whereas those from rats with the glutamate-loaded nanofiber mat dressing showed many intraparenchymal microhemorrhages of variable sizes. The capillaries in the vicinity of the glutamate-carrying dressing (in the meninges and white matter alike) were edematous and leaky, and their endothelial cells showed degenerative changes: extensive swelling, enhanced vacuolization and the presence of vascular intraluminal projections. However, endothelial tight junctions were generally well preserved. Some endothelial cells were dying by necrosis or apoptosis. The adjacent parenchyma showed astrogliosis with astrocytic hypertrophy and swelling of perivascular astrocytic feet. Neurons in the parenchyma revealed multiple symptoms of degeneration, including, inter alia, perikaryal, dendritic and axonal swelling, and destruction of organelles. All the damage symptoms were slightly less severe in the rats given valproic acid treatment, and were absent from both the intact rats and the rats with 'empty' nanofiber mat dressing. These results demonstrate that glutamate-loaded nanofiber mat dressing can locally create glutamate levels capable of damaging BSCB and that the resulting damage can be mitigated with concurrent systemic valproate treatment.
\end{abstract}

Key words: astrocyte, blood-spinal cord barrier, CNS damage, degeneration, endothelium, excitotoxicity, glutamate, neuron, valproate, vessels. 


\section{Introduction}

The blood-brain barrier (BBB) and blood-spinal cord barrier (BSCB) are composite structures the role of which is selective separation of the cerebral and spinal parenchyma from the contents of the blood vessel; these barriers play both a protective and regulatory role for the respective central nervous system (CNS) parenchyma [3]. The blood-brain barrier and $\mathrm{BSCB}$ are prone to damage from insults of varying origin, including local inflammatory processes, ischemia, hypoxia, mechanical trauma, and more.

The two barriers slightly differ structurally and functionally, mostly in ultrastructure of their endothelial cells $[8,12,32,34,40]$. These differences may be responsible for diverse susceptibility of BBB and $\mathrm{BSCB}$ to some pathological conditions [3]. Many authors suggest that disruption of BSCB or alteration of its permeability may be of key importance for the consequences of acute injury of the spinal cord [47] and for the development of some CNS pathologies, such as multiple sclerosis [37], amyotrophic lateral sclerosis [15,29], spinal cord ischemia $[16,18,27]$, and neuropathic pain [4].

The main components of BSCB are endothelial cells, basement membrane and pericytes; some role is also played by perivascular astrocytes, the processes of which envelop small blood vessels $[3,9]$. An important role of astrocytes in the CNS is to support neurons under both normal physiological and pathological conditions, both by metabolic and regulatory means $[10,25,44,46]$. Due to the presence of active uptake and/or metabolic systems for some monoamine and amino acid neurotransmitters they can also contribute to the regulation of their interstitial levels $[19,20,39]$. After various CNS insults, astroglial cells invade the damaged region, become activated and hypertrophic, and, inter alia, start to express and release multiple trophic factors, including chemokines and cytokines [13].

An excessive glutamate level can cause damage and death of CNS cells and is also known as an important contributing factor in many CNS disorders $[21,33]$. Glutamate-damaged neurons show definite morphological alterations. Excitotoxic neuronal injury may correlate with BBB damage [7].

There is an ongoing search for new approaches to delivering various drugs into the CNS, and in particular those capable of chronic delivery. Our earlier data have shown the usefulness of subarachnoidal elec- trospun nanofiber mat dressing as the drug delivery system capable of long-term release of glutamate levels sufficient for damaging spinal cord motoneurons in the rat [36]. The aim of the present investigation was to examine the effects of this treatment on morphology of the various components of the BSCB, especially on endothelial cells.

\section{Material and methods \\ Nanofiber mats}

Drug-free ('empty') electrospun nanofiber mats as well as monosodium glutamate (MSG)-loaded electrospun nanofiber mats were prepared as described earlier [36].

\section{Animals and experimental design}

Adult male Wistar rats (starting body weight 250$300 \mathrm{~g}, n=12$ ) from the animal facility of the Mossakowski Medical Research Centre were used for the study. The rats were kept in three per opaque plastic cage $(55 \times 33 \mathrm{~cm}$ floor size) in an air-conditioned $\left(60-70 \%\right.$ relative humidity, $\left.21 \pm 2^{\circ} \mathrm{C}\right)$ room at a $12 \mathrm{~h}$ light/12 h dark day cycle (lights on at 7 a.m.), and were given free access to sterilized standard laboratory rat maintenance chow (Ssniff, Soest, Germany) and tap water.

The rats were randomized between four experimental groups of three rats each: 1) intact controls (group I), 2) rats with drug-free nanofiber mat application into the spinal cord subarachnoid space at the lumbar enlargement level (group II), 3) rats with MSG-loaded nanofiber mat (14.2 mg MSG/mg of the mat) into the subarachnoid space (group III); and 4) rats subjected to a surgical placement of the same glutamate-loaded mat into the subarachnoid space, which were additionally given by gavage one dose of the histone deacetylase inhibitor sodium valproate (Convulex syrup, $50 \mathrm{mg} / \mathrm{ml}$, Gerot Pharmazeutika, Wien, Austria) daily, beginning on the surgery day (group IV). The initial valproate dose was $33.3 \mathrm{mg} / \mathrm{kg}$ body weight; the dosage was elevated by $8.3 \mathrm{mg} / \mathrm{kg}$ each day for 4 days and then was kept at $67 \mathrm{mg} / \mathrm{kg}$ body weight for the remainder of the study period.

Subarachnoidal implantation of nanofiber mat pieces $(5 \times 5 \mathrm{~mm}$ size) into rat spinal cords (at the lumbar enlargement level) was performed as described earlier [1]. Three weeks after the surgery, all rats were deeply anesthetized with pentobarbital (80 mg/kg, i.p.) and decapitated. The L1-L6 segments 
of their spinal cords were harvested and immediately fixed in $4 \%$ formaldehyde solution in PBS and next embedded in paraffin by a standard procedure. The paraffin blocks were then cut and processed for histological and immunohistochemical methods.

All animal use procedures were in agreement with the European Communities Council Directive on the protection of laboratory animals (86/609/ EEC) and with the current Polish law. All efforts were made to reduce animal discomfort and the number of rats used. The protocol of the study has been accepted by the $4^{\text {th }}$ Local Animal Experimentation Ethics Committee at the National Medicines Institute, Warsaw, Poland (Certificate No. 43/210).

\section{Histology and immunohistochemistry}

The paraffin-embedded spinal cord samples were cut crosswise into $8 \mu \mathrm{m}$ sections. After deparaffinization and rehydration in a water-ethanol solution series by standard procedures, some of the sections were stained with hematoxylin-eosin mixture solution using a routine procedure. The sections selected for immunohistochemistry were first incubated with rabbit polyclonal anti-GFAP antibody (Dako cat. no. Z0334; diluted 1 : 4000). Next, the sections were incubated with goat anti-rabbit antibody (Beckman Coulter Inc., France, cat. no. IM0830; dil. 1 : 100) and then with streptavidin-horseradish peroxidase solution (Beckman Coulter cat. no. IM0309; dil. 1 : 500). The resulting immune complexes were visualized by a routine procedure with diaminobenzidine as the chromogen and then counterstained with hematoxylin. The intensity of GFAP immunostaining was assessed by an experimenter blinded to the samples identity, using a light microscope (Nikon, Japan) equipped with a CCD camera and a PC-based image analyzer system. Specificity of the staining was tested by running the same procedure on the respective sister sections with the primary antibody absent from the incubation mixture; the control sections revealed no immunosignal.

\section{Transmission electron microscopy}

Samples of lumbar spinal cord meant for electron microscopy were instantly fixed in a formaldehyde-glutaraldehyde ( $2 \% / 2.5 \%)$ solution in cacodylate buffer $\mathrm{pH} 7.4$ for $4 \mathrm{~h}$. Next, they were cut into smaller fragments, rinsed in the cacodylate buffer, post-fixed in $1.0 \% \mathrm{OsO}_{4}$ solution for $1 \mathrm{~h}$, dehydrat- ed by a standard procedure, embedded in epon resin, and cut into $40 \mu \mathrm{m}$-thick sections. The sections were stained with $1 \%$ toluidine blue and examined in a light microscope for block selection. The selected blocks were then cut into ultrathin sections that were stained with uranyl acetate and lead citrate and then examined with a model JEOL 1200EX (Jeol, Japan) electron microscope by an experimenter blinded to the samples identity.

\section{Results}

\section{Light microscopy morphological and immunohistochemistry studies}

Spinal cords from the intact rats and rats carrying 'empty' nanofiber mat dressing showed normal parenchyma and BSCB morphology. Spinal cords from the rats carrying MSG-loaded spinal cord dressing (group III) revealed the presence of multiple intraparenchymal microhemorrhages of varying sizes (Figs. 1 and 2). The capillaries in the vicinity of the dressing (both in the meninges and the white matter) were leaky and showed considerable swelling; there was no difference in these characteristics between rats with and without systemic valproic acid treatment (group IV and group III, respectively).

Immunohistochemistry revealed astrogliosis, presence of numerous activated hypertrophic astrocytes (Fig. 3) and swelling of the astroglial end-feet processes located close to the leaky vessels both in group III and group IV; however, the symptoms were more severe in group III. No signs of astroglial reaction were found in the control group with 'empty' subarachnoidal nanofiber mat dressing (Fig. 3).

\section{Electron microscopy studies}

In the intact controls (group I) and in the rats with 'empty' nanofiber mat dressing (group II) (Fig. 4), electron microscopy showed proper morphology of blood vessels, neurons and glial cells. The structure of microvascular basement membrane was homogenous and tight (Figs. 2-4); the vicinity of the vessels showed the presence of normal-looking astrocytic end-feet and scanty intercellular space.

In group III rats, neurons revealed both cytoplasmic and mitochondrial edema, organelle decay, endoplasmic reticulum atrophy and cytoplasmic microvacuolization. These alterations were more extensive in postsynaptic dendrites (Fig. 5A) than in neuronal perikarya (Fig. 5B). Similar changes were seen in the cyto- 

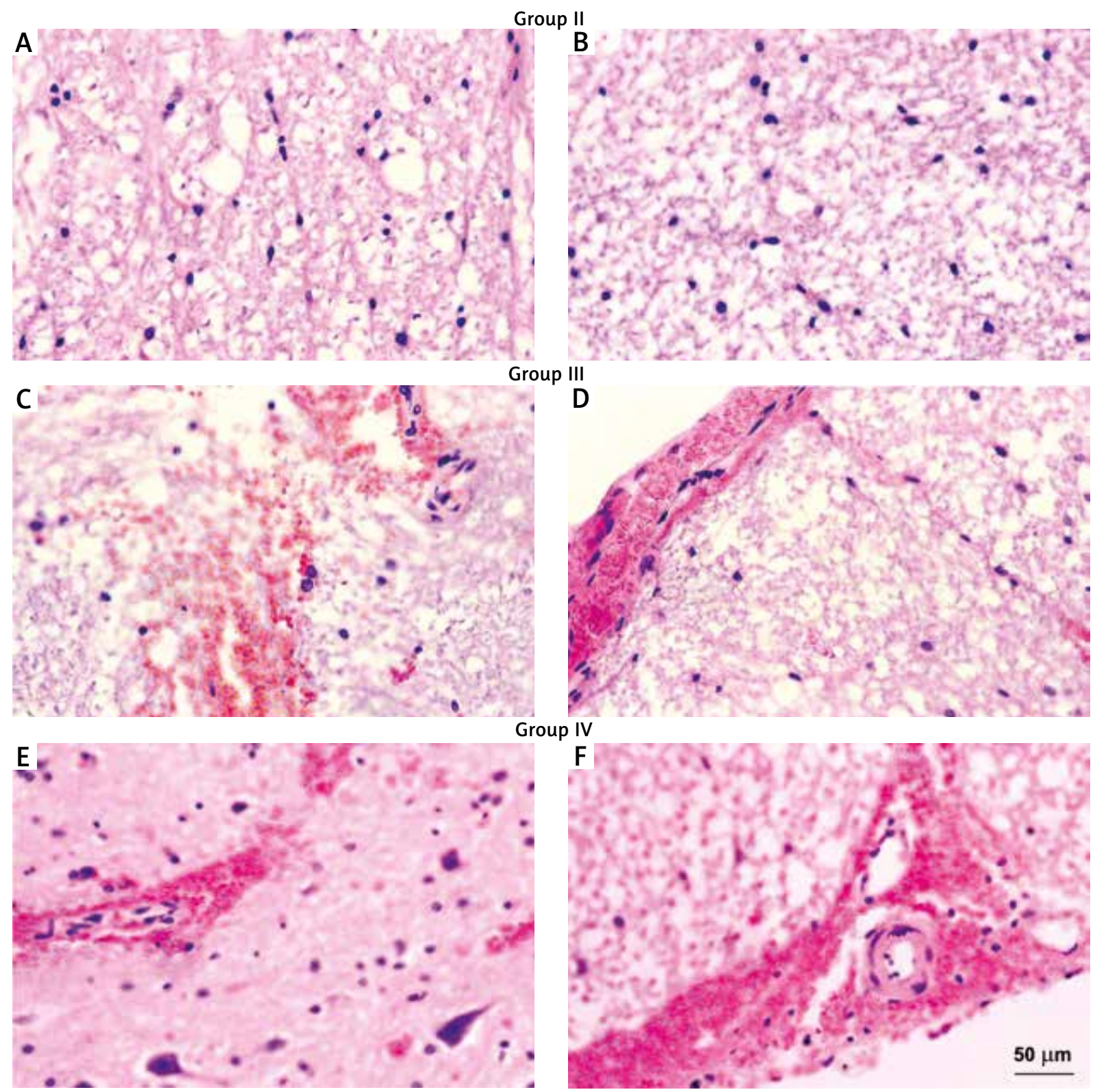

Fig. 1. Microhemorrhages in the vicinity of the nanofiber mat dressing (hematoxylin-eosin staining). The spinal cord section from a group II rat (control with 'empty' mat dressing) shows no visible microhemorrhages in the parenchyma. The sections from a group III rat and a group IV rat show the presence of microhemorrhages both within the parenchyma (left column) and in the subarachnoid space (right column).

plasm of both astroglial cells (Fig. 5C), small vessel endothelial cells and pericytes (Fig. 5D). The vessels showed endothelial cells damage of varying intensity and thickened basement membrane; edema of perivascular end-feet processes and neuropil, and expansion of the interstitial space were apparent as well. Some vessels showed endothelial cells necrosis with destruction of cell membrane and deterioration of tight junctions (Figs. 5B and 6A). A typical pattern of apoptotic changes of the endothelium was but rarely seen (Fig. 6B). More frequently, the endothelium showed a mixed pattern of overlapping apoptotic and necrotic changes (Fig. 6C-D), with increased cytoplasm density and relatively well-preserved organelles in some (shrunken) cells, and cytoplasmic edema and vacuolization, organelle and cell membrane decay in the other cells. Most of the damaged endothelial cells had an irregular luminal surface, with multiple 


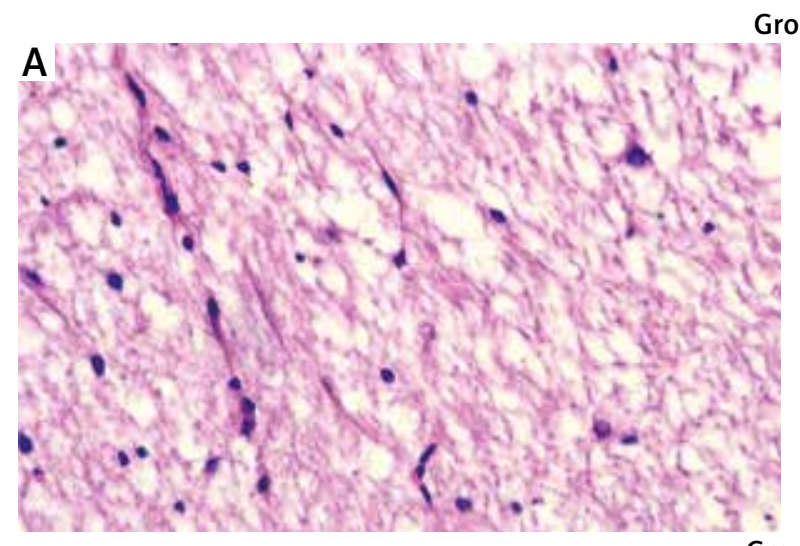

Group II

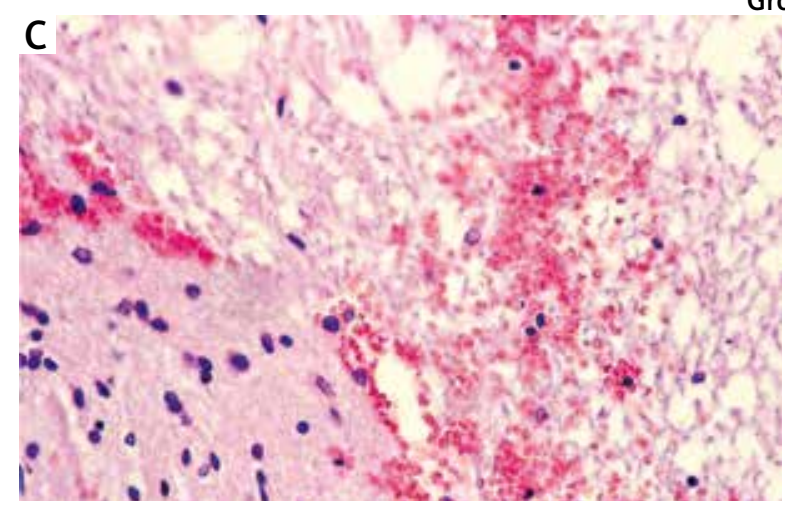

Group III
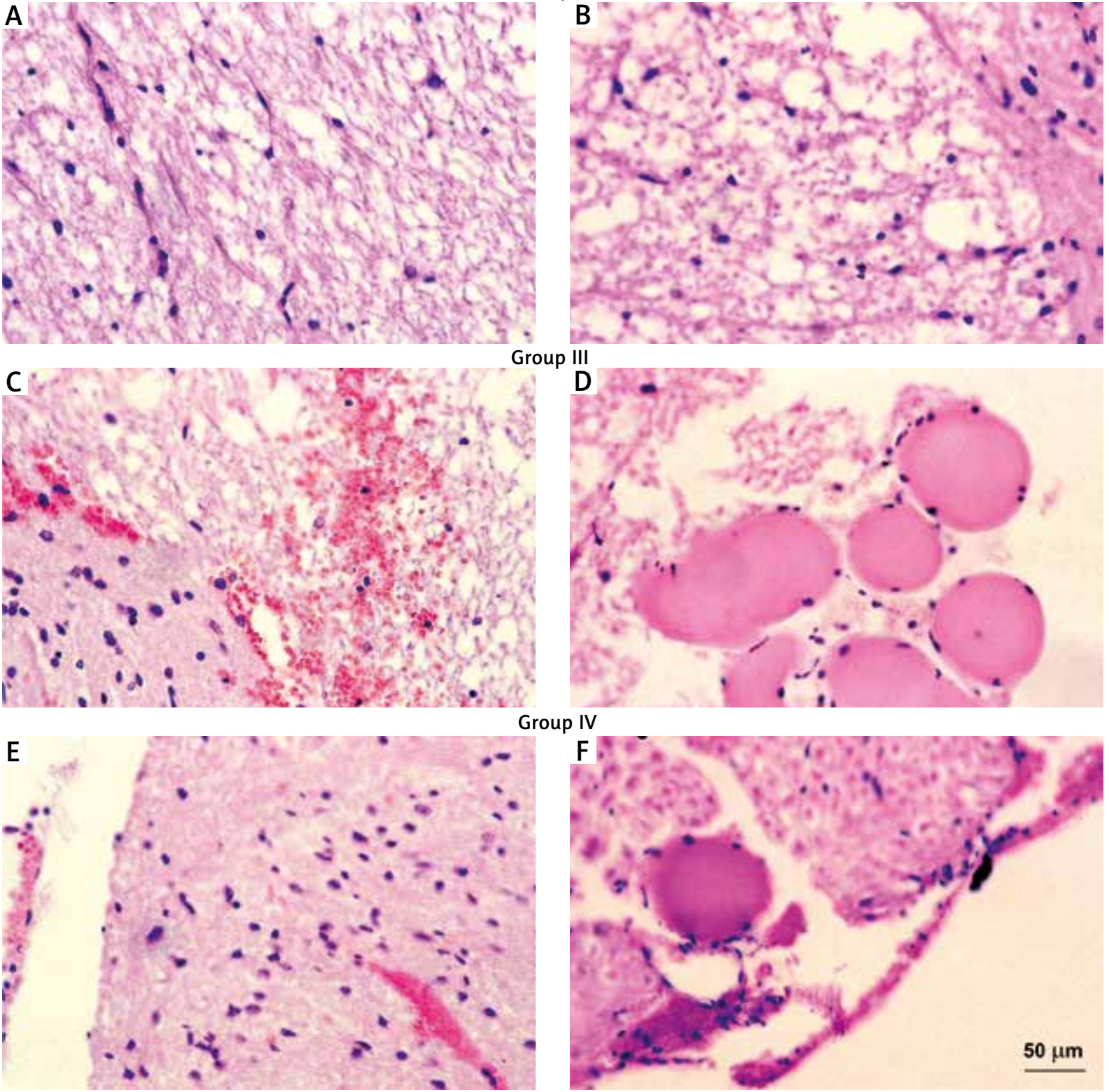

Group IV
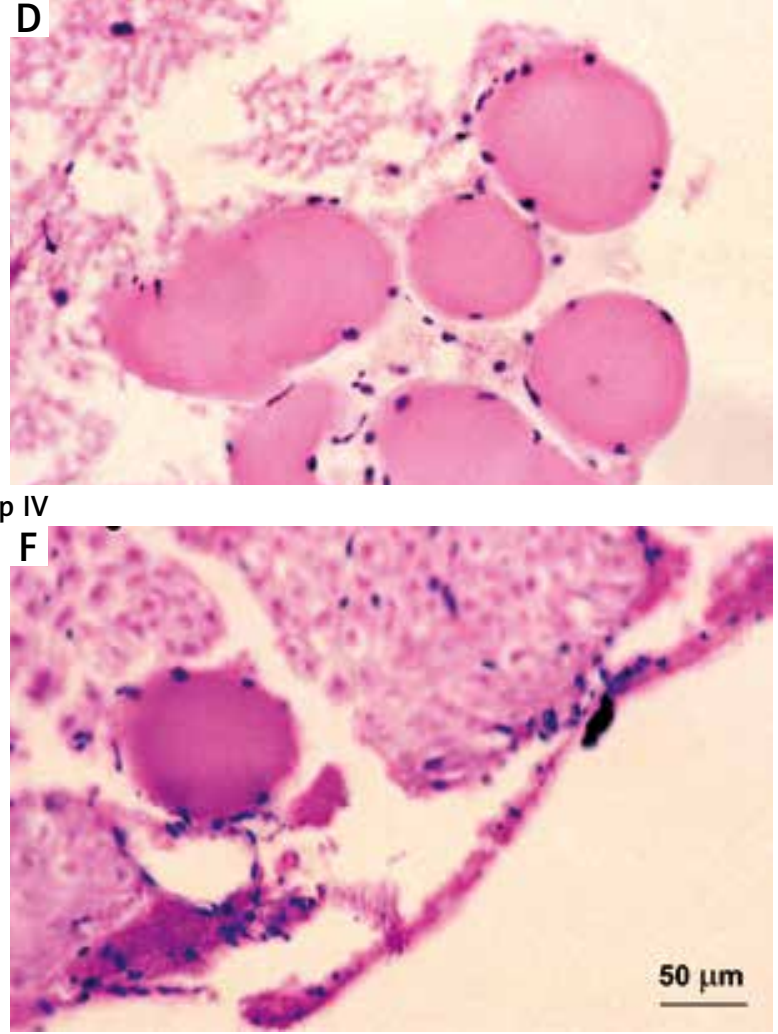

Fig. 2. Microhemorrhages (left column) and dilation of spinal cord capillary vessels (right column) in the vicinity of the nanofiber mat dressing (hematoxylin-eosin staining). The section from a group II (control) rat shows no vessel dilation. The sections from group III and group IV rats show the presence of dilated capillaries/microvessels in the parenchyma, meninges and posterior funicles.

intraluminal cytoplasmic microvilli-like protrusions, increased numbers of plasmalemmal vesicles, cytoplasmic vacuoles and irregular endoplasmic reticulum channels (Fig. 6C-E). Most endothelial tight junctions remained closed (Fig. 6E); opened tight junctions were occasionally seen, especially in the proximity of the basement membrane. The basement membrane of the vessels was mostly thickened and stratified, with embedded fragments of pericytes (Fig. 6E).
The adjacent parenchyma showed considerable edema and decay of neuropil and astrocytic perivascular processes (Fig. 6C-D).

In the spinal cords of group IV rats, the edema of the endothelium and perivascular astrocytic end-feet was of clearly lesser severity (Figs. 7A-B); the endothelial cells showed nearly normal luminal cellular membrane (with only occasional intraluminal protrusions), mostly well-preserved mitochondria, ribo- 

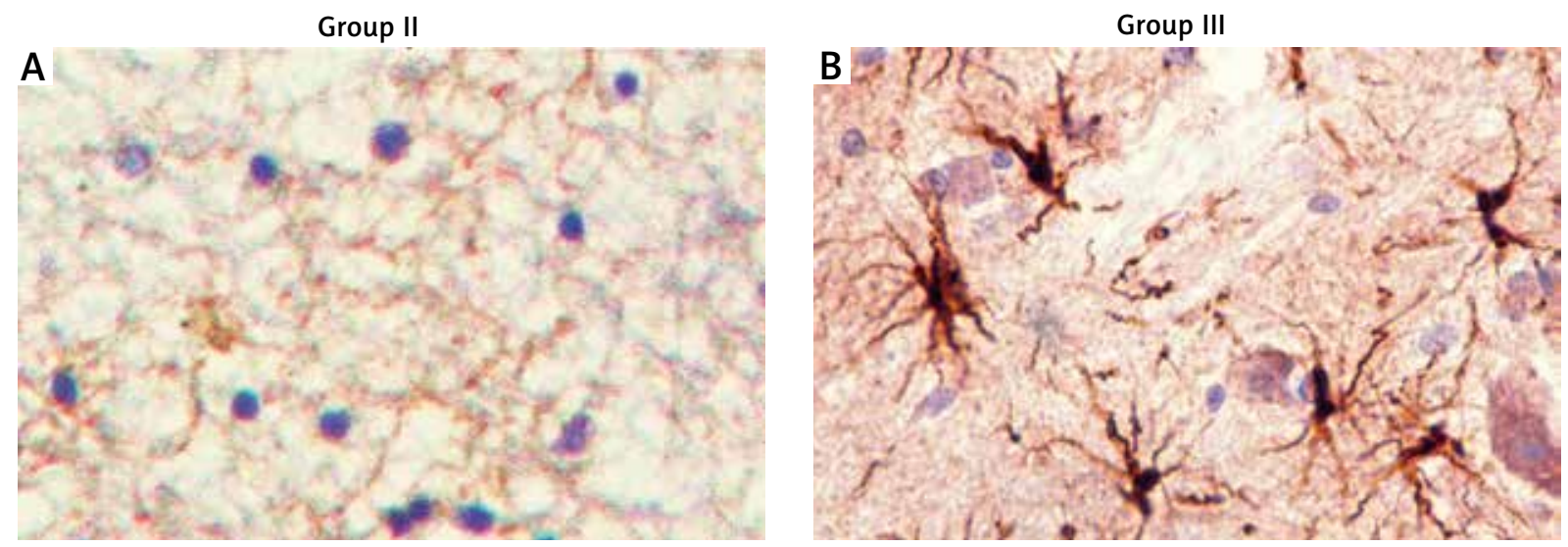

Group IV

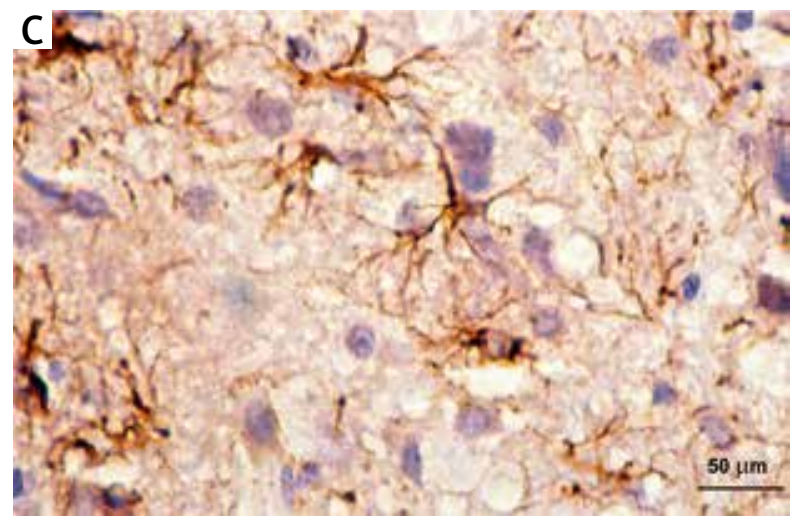

Fig. 3. Perivascular astrocytes of spinal cord capillary vessels in the vicinity of the nanofiber mat dressing (GFAP immunostaining). The spinal cord section from a group II rat shows astroglia with normal morphology. The spinal cord section from a group III rat shows activation (hypertrophy of perikarya and processes) in a majority of astroglial cells, and clasmatodendrosis (signs of degeneration) in some astrocytes. The spinal cord section from a group IV rat shows astroglial activation of lesser severity and no visible clasmatodendrosis.

somes and endoplasmic reticulum; single autophagic vacuoles were but sporadically seen. In summary, all the MSG-loaded electrospun nanofiber mat dressing-induced alterations were more severe in group III than in group IV rats.

\section{Discussion}

Glutamate belongs to the most extensively studied neurotransmitters. Its physiological content in the nervous tissue corresponds with the metabolic demand of the cells, and the BBB and BSCB protect CNS parenchyma from an influx of excessive extraCNS glutamate amounts [26]. It is common knowledge that CNS glutamate concentration is greatly elevated in many CNS pathologies $[23,35,43,48]$. It has been demonstrated as well that the considerable ischemia-related elevation of intracerebral glutamate results in BBB damage and brain edema [41].
Glutamate is also assigned an important role in the pathomechanisms of many CNS pathologies, e.g., of epilepsy, ischemia and amyotrophic lateral sclerosis, and in acute brain or spinal cord injury $[2,26,28,30]$.

The present study is a continuation of our earlier investigation that demonstrated a toxic effect of exogenous glutamate delivered by subarachnoidal application of MSG-loaded electrospun nanofiber mat dressing on spinal cord motoneurons and a neuroprotective action of concurrent sodium valproate treatment [36]. This study was aimed at determining the potential of subarachnoidal electrospun nanofiber mat dressing for delivering MSG in the amount sufficient for inducing BSCB damage, and at defining the effect of the exogenous glutamate on perivascular cells of the spinal cord parenchyma. We have demonstrated that the chronic action of the mat-released glutamate damages BSCB. Blood 

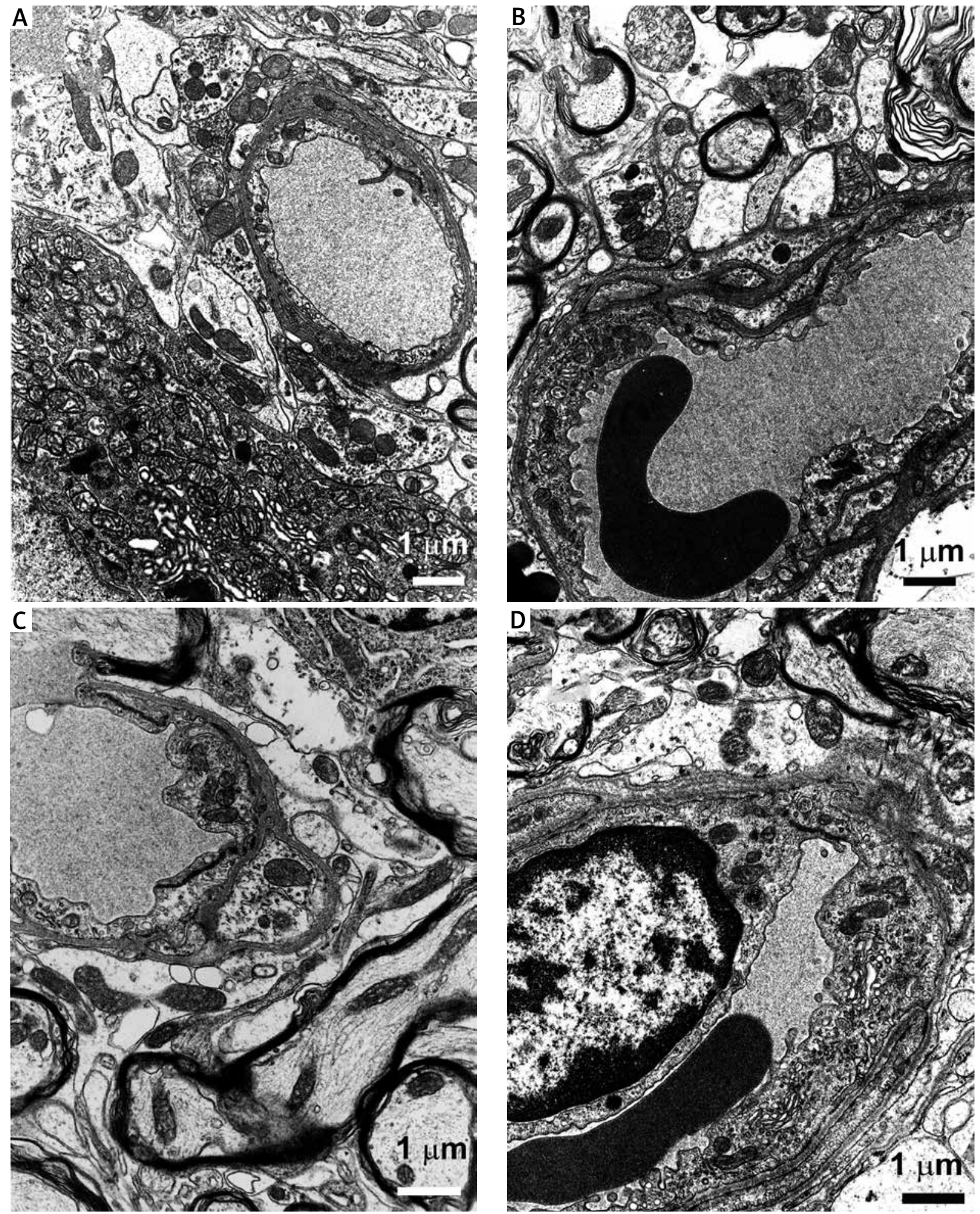

Fig. 4. Representative electron micrographs of spinal cord sections from a group II rat. A) Well-preserved organelles, e.g. mitochondria, are visible both in neuronal cytoplasm and in processes of neuronal, glial as well as endothelial cells of a normal-looking capillary. B-C) Microvessels with normal ultrastructure, smooth luminal surface of the endothelium and well-preserved endothelial tight junctions (upper left corner). The cytoplasm of endothelial cells and pericytes shows the presence of small dark mitochondria, normal-looking endoplasmic reticulum and few vacuoles. D) The endothelial nucleus has clear euchromatin and evenly distributed heterochromatin. 

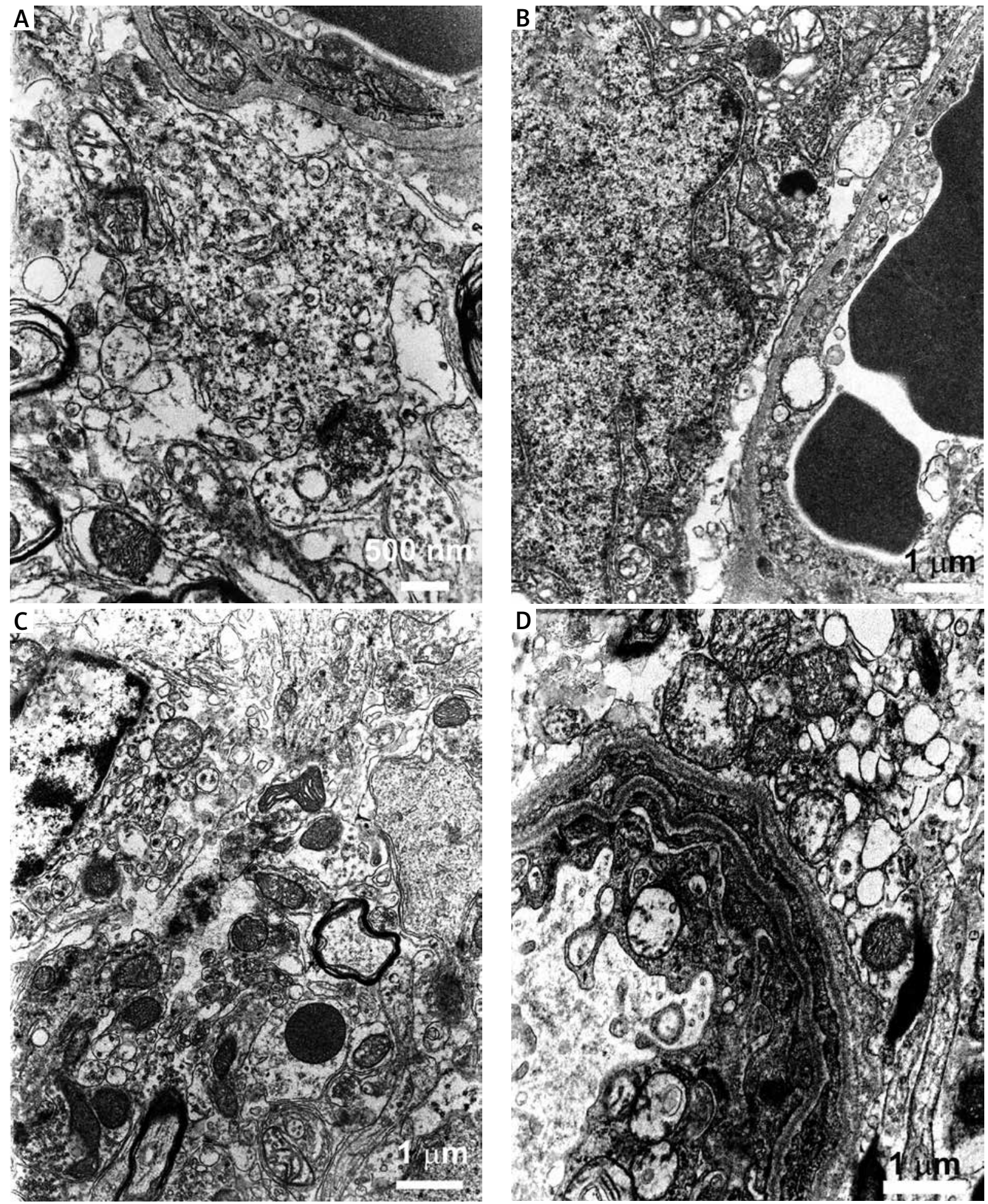

Fig. 5. Representative electron micrographs of spinal cord sections from a group III rat showing degenerative changes. A) Neuron showing decay of organelles, dendrite degeneration, and microvacuolization. Perivascular parenchyma is swollen. B) Neurons show perikaryal degeneration as well as edema and decay of organelles. In the lower right corner of the photograph, a fragment of a necrotic endothelial cell is visible, which shows destruction of cell membrane. C) Degeneration and decay of astrocytic organelles. D) Degenerative alterations in a perivascular astrocyte and capillary endothelium. 

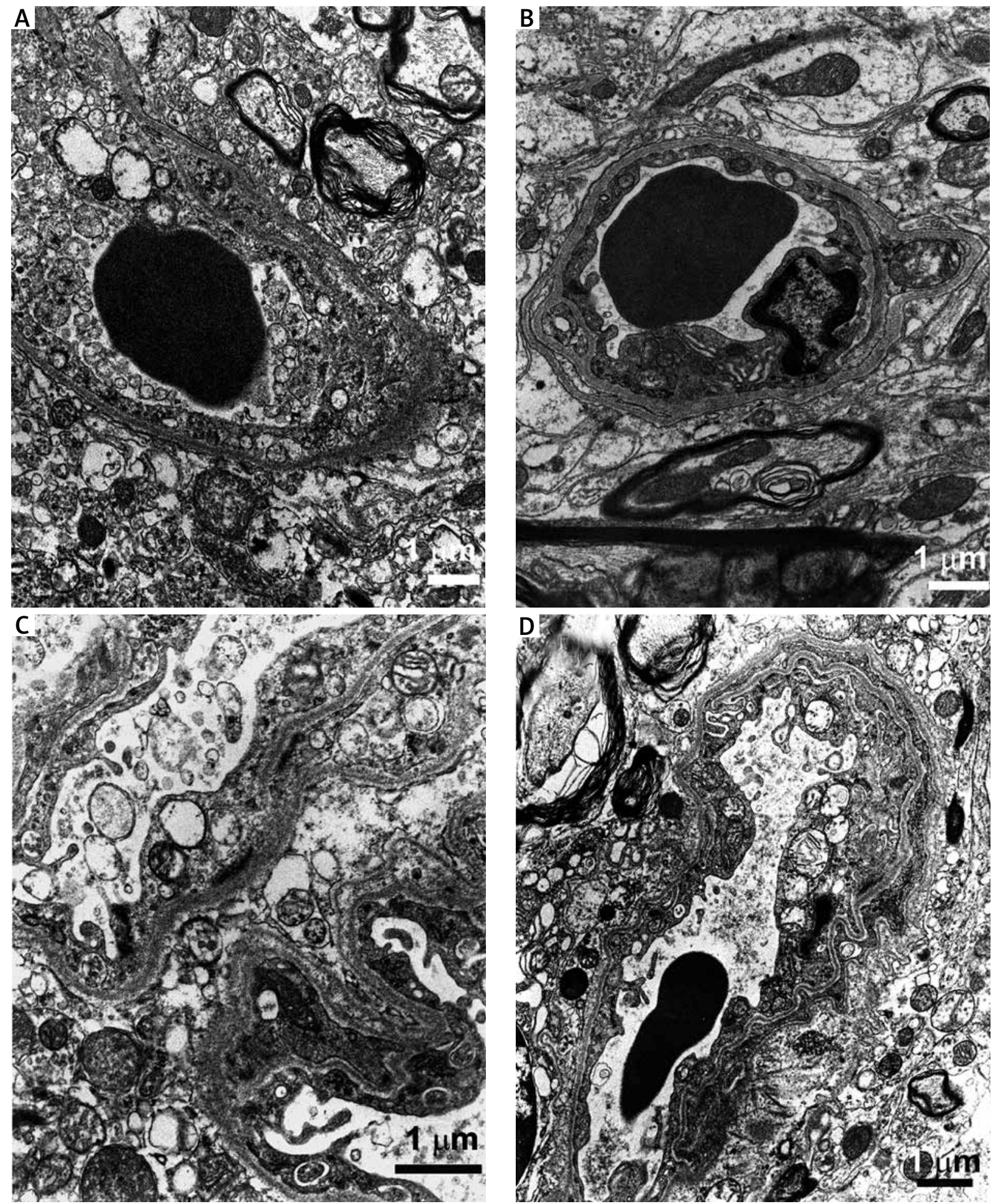

Fig. 6. Representative electron micrographs of spinal cord sections from a group III rat showing cell death. A) Damage of cell membrane in capillary endothelium. B) Early apoptotic changes in an endothelial cell. C-D) Coexistence of apoptotic and necrotic cells. Perivascular zone shows decay of the neuropil and astrocytic processes. 


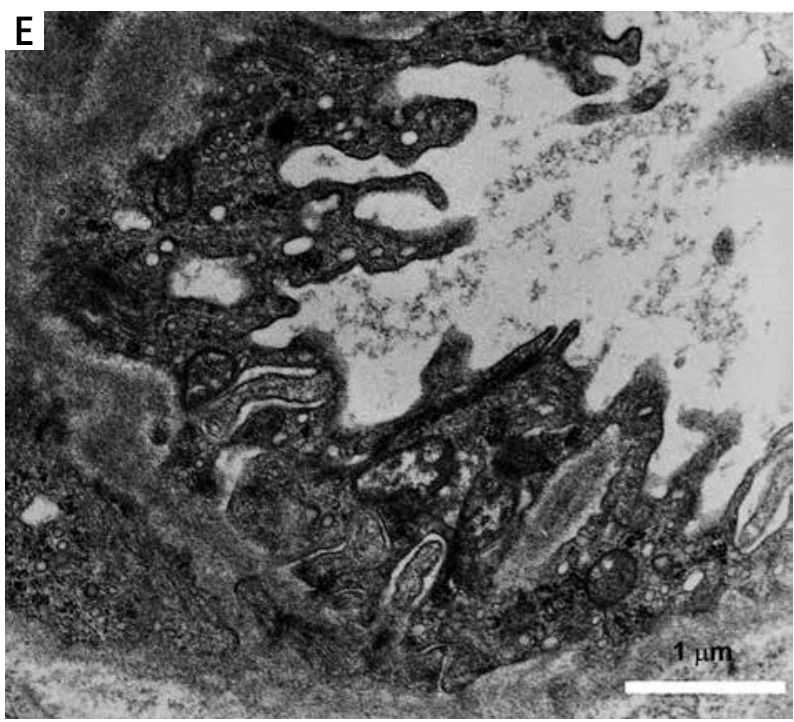

Fig. 6 cont. Representative electron micrographs of spinal cord sections from a group III rat showing cell death. E) Thickened basement membrane with visible fragment of a pericyte. Capillary endothelium shows the presence of multiple microvacuoles and carries intraluminal microvilli-like processes.
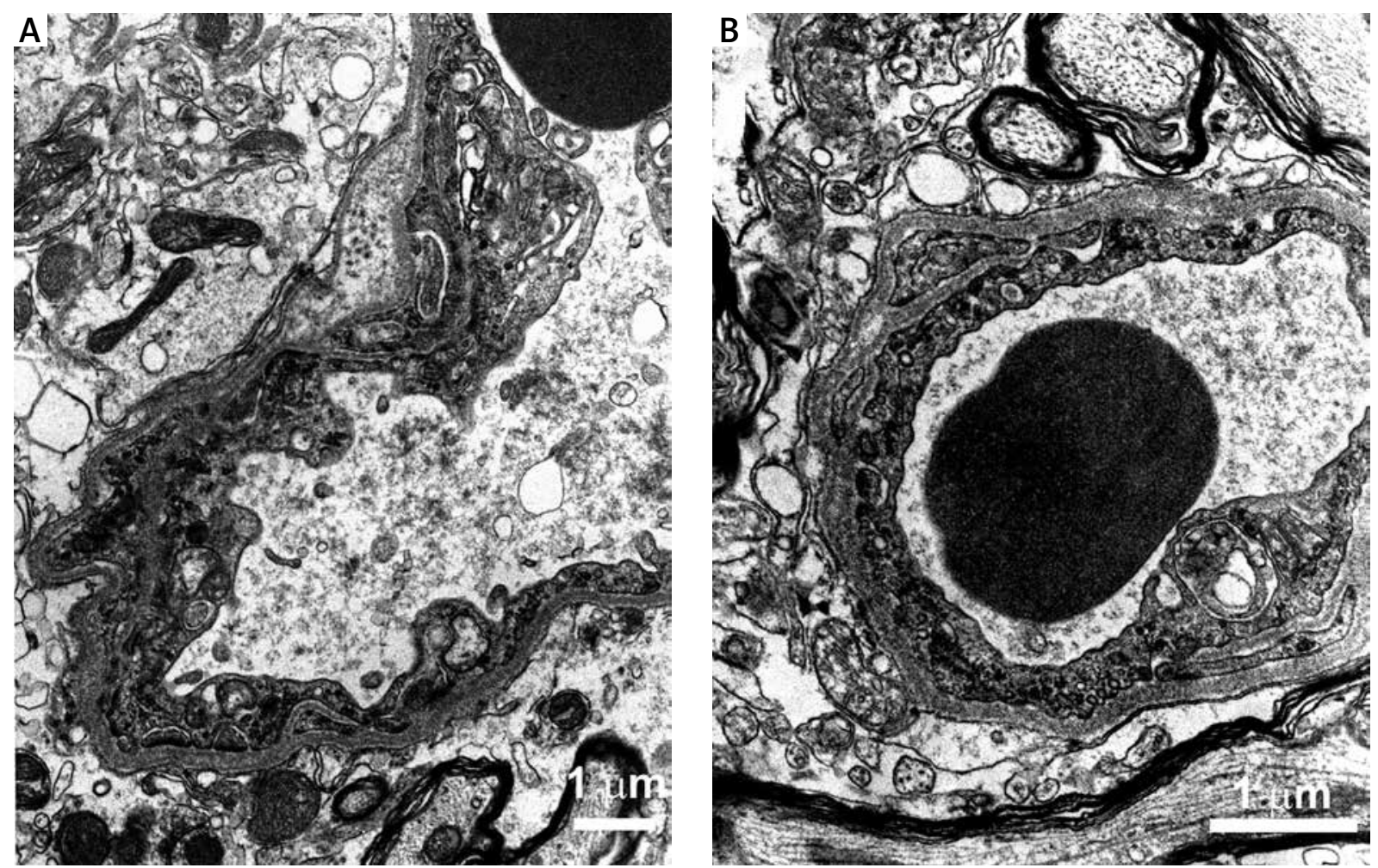

Fig. 7. Representative electron micrographs of spinal cord sections from a group IV rat. A-B) Moderate edema of vascular endothelium and perivascular astrocyte end-feet processes. Endothelial cells show almost smooth luminal cell membrane, mostly well-preserved organelles and considerably less cytoplasmic microvacuoles than in the group III (Fig. 6E) rat. 
vessels become dilated and permeabilized, and their vicinity showed the presence of edema and multiple microhemorrhages. Our studies have also revealed a strong astrocytic reaction in the vicinity of the damaged blood vessels, which demonstrated itself in marked astrogliosis and cell activation. However, some of the astrocytes showed clasmatodendrosis evidencing cell degeneration. Electron microscopy has also demonstrated the presence of 'empty' astrocytic end-feet processes evidencing perivascular edema. Considerable alterations were also visible in the cytoplasm and organelles of endothelial cells and in the adjacent basement membrane. The vicinity of the vessels showed also the presence of degenerating neurons. Interestingly, the glutamate-evoked alterations of the BSCB did not significantly affect the state of these cells in that their condition, while poor, was not worse than that of the neurons more distal to the damaged vessels [36]. It is believed that the basis of the glutamate-induced cell damage are, inter alia, an enhanced generation of free radicals and excessive calcium ions levels [11,13,14,17,38,45], which ultimately cause permeabilization of blood vessel walls by damaging BBB/BSCB integrity via a variety of signal transduction pathways and/or by direct action on endothelial tight junctions [6].

Glutamate excitotoxicity causes severe disturbances in protein and lipid metabolism by activating AMPA, NMDA and kainate glutamate receptors $[24,26]$, and in particular the endothelial NMDA receptor subset $[5,22]$. Excessive stimulation of the NMDA receptors causes imbalance of $\mathrm{Na}^{+}$ions across plasma membranes [31], increased influx of $\mathrm{Ca}^{2+}$ ions and activation of a wide spectrum of intracellular enzymes, including kinases, proteases and phospholipases [26], and promotes free radical-related oxidative stress in endothelial cells [42]. The latter in turn causes activation of NMDA receptors, resulting in forming of a metabolic vicious circle that intensifies functional and structural disturbances of cell membranes and increases BBB/BSCB permeability.

All said, the results of this study indicate that MSG-loaded nanofiber mat dressing can create glutamate concentrations capable of damaging both the $\mathrm{BSCB}$ and the neighboring parenchyma in the rat. Whereas the glutamate released from the dressing damages them both considerably, the concurrent systemic administration of the histone deacetylase inhibitor valproic acid alleviates the detrimental consequences of the long-term action of excessive glu- tamate levels on parenchyma more than those seen in the barrier.

\section{Acknowledgments}

This work was supported by grant no. NN 401 014640 from the Ministry of Science and Higher Education of Poland, and by statutory funds from the Mossakowski Medical Research Centre, Polish Academy of Sciences. The authors thank Dr. Tomasz Kowalczyk of the Institute of Fundamental Technological Research, Polish Academy of Sciences, for his consulting the preparation and in vitro characterization of the electrospun nanofiber mats.

\section{Disclosure}

Authors report no conflict of interest.

\section{References}

1. Andrychowski J, Frontczak-Baniewicz M, Sulejczak D, Kowalczyk T, Chmielewski T, Czernicki Z, Kowalewski TA. Nanofiber nets in prevention of cicatrization in spinal procedures. Experimental study. Folia Neuropathol 2013; 51: 147-157.

2. Barker-Haliski M, White HS. Glutamatergic mechanisms associated with seizures and epilepsy. Cold Spring Harb Perspect Med 2015; 5: a022863.

3. Bartanusz V, Jezova D, Alajajian B, Digicaylioglu M. The bloodspinal cord barrier: morphology and clinical implications. Ann Neurol 2011; 70: 194-206.

4. Beggs S, Liu XJ, Kwan C, Salter MW. Peripheral nerve injury and TRPV1-expressing primary afferent C-fibers cause opening of the blood-brain barrier. Mol Pain 2010; 6: 74.

5. Betzen C, White R, Zehendner CM, Pietrowski E, Bender B, Luhmann HJ, Kuhlmann CR. Oxidative stress upregulates the NMDA receptor on cerebrovascular endothelium. Free Radic Biol Med 2009; 47: 1212-1220.

6. Brown RC, Davis TP. Calcium modulation of adherens and tight junction function: a potential mechanism for blood-brain barrier disruption after stroke. Stroke 2002; 33: 1706-1711.

7. Chen ZL, Indyk JA, Bugge TH, Kombrinck KW, Degen JL, Strickland S. Neuronal death and blood-brain barrier breakdown after excitotoxic injury are independent processes. J Neurosci 1999; 19: 9813-9820.

8. Daniel PM, Lam DK, Pratt OE. Changes in the effectiveness of the blood-brain and blood-spinal cord barriers in experimental allergic encephalomyelitis. J Neurol Sci 1981; 52: 211-219.

9. Engelhardt B, Sorokin L. The blood-brain and the blood-cerebrospinal fluid barriers: function and dysfunction. Semin Immunopathol 2009; 31: 497-511.

10. Fawcett JW, Asher RA. The glial scar ad central nervous system repair. Brain Res Bull 1999; 49: 377-391.

11. Fern R, Ransom BR, Waxman SG. Voltage-gated calcium channels in CNS white matter: role in anoxic injury. J Neurophysiol 1995; 74: 369-377. 
12. Ge S, Pachter JS. Isolation and culture of microvascular endothelial cells from murine spinal cord. J Neuroimmunol 2006; 177: 209-214

13. Hausmann ON. Post-traumatic inflammation following spinal cord injury. Spinal Cord 2003; 41: 369-378.

14. Hazell AS. Excitotoxic mechanisms in stroke: An update of concepts and treatment strategies. Neurochem Int 2007; 50: 941 953.

15. Henkel JS, Beers DR, Wen S, Bowser R, Appel SH. Decreased mRNA expression of tight junction proteins in lumbar spina cords of patients with ALS. Neurology 2009; 72: 1614-1616.

16. Jacobs TP, Kempski O, McKinley D, Dutka AJ, Hallenbeck JM, Feuerstein G. Blood flow and vascular permeability during motor dysfunction in a rabbit model of spinal cord ischemia. Stroke 1992; 23: 367-373.

17. Jiang R, Diaz-Castro B, Looger LL, Khakh BS. Dysfunctional calcium and glutamate signaling in striatal astrocytes from Huntington's disease model mice. J Neurosci 2016; 36: 3453-3470.

18. Kaur C, Ling EA. Blood brain barrier in hypoxic-ischemic conditions. Curr Neurovasc Res 2008; 5: 71-81.

19. Kimelberg HK, Katz DM. High-affinity uptake of serotonin into immunocytochemically identified astrocytes. Science 1985; 228: 889-891.

20. Kimelberg HK, Norenberg MD. Astrocytes. Sci Am 1989; 260: 44-52.

21. Kostandy BB. The role of glutamate in neuronal ischemic injury: the role of spark in fire. Neurol Sci 2012; 33: 223-2237.

22. Krizbai IA, Deli MA, Pestenácz A, Siklós L, Szabó CA, András I, Joó F. Expression of glutamate receptors on cultured cerebral endothelial cells. J Neurosci Res 1998; 54: 814-819.

23. Kuhlmann CRW, Gerigk M, Bender B, Closhen D, Lessmann V, Luhmann HJ. Fluvastatin prevents glutamate-induced bloodbrain-barrier disruption in vitro. Life Sci 2008; 82: 1281-1287.

24. LeMaistre JL, Sanders SA, Stobart MJ, Lu L, Knox JD, Anderson HD, Anderson CM. Coactivation of NMDA receptors by glutamate and D-serine induces dilation of isolated middle cerebral arteries. J Cereb Blood Flow Metab 2012; 32: 537-547.

25. Magistretti PJ, Pellerin L. Astrocytes couple synaptic activity to glucose utilisation in the brain. News Physiol Sci 1999; 14: 177-182.

26. Mallick HN. Understanding safety of glutamate in food and brain. Indian J Physiol Pharmacol 2007; 51: 216-234.

27. Matsumoto S, Matsumoto M, Yamashita A, Ohtake K, Ishida K, Morimoto Y, Sakabe T. The temporal profile of the reaction of microglia, astrocytes, and macrophages in the delayed onset paraplegia after transient spinal cord ischemia in rabbits. Anesth Analg 2003; 96: 1777-1784.

28. Meldrum BS. Glutamate as a neurotransmitter in the brain: review of physiology and pathology. J Nutr 2000; 130 (4S Suppl): 1007S-1015S

29. Miller RG, Jackson CE, Kasarskis EJ, England JD, Forshew D, Johnston W, Kalra S, Katz JS, Mitsumoto H, Rosenfeld J, Shoesmith C, Strong MJ, Woolley SC; Quality Standards Subcommittee of the American Academy of Neurology. Practice parameter update: the care of the patient with amyotrophic lateral sclerosis: multidisciplinary care, symptom management, and cognitive/ behavioral impairment (an evidence-based review): report of the Quality Standards Subcommittee of the American Academy of Neurology. Neurology 2009; 73: 1227-1233.

30. Nasser M, Bejjani F, Raad M, Abou-El-Hassan H, Mantash S, Nokkari A, Ramadan N, Kassem N, Mondello S, Hamade E, Darwish H, Zibara K, Kobeissy F. Traumatic brain injury and bloodbrain barrier cross-talk. CNS Neurol Disord Drug Targets 2016; 15: $1030-1044$.

31. Nishizawa Y. Glutamate release and neuronal damage in ischemia. Life Sci 2001; 69: 369-381.

32. Pan W, Banks WA, Kastin AJ. Permeability of the blood-brain and blood-spinal cord barriers to interferons. J Neuroimmunol 1997; 76: 105-111.

33. Platt SR. The role of glutamate in central nervous system health and disease - a review. Vet J 2007; 173: 278-286.

34. Prockop LD, Naidu KA, Binard JE, Ransohoff J. Selective permeability of [3H]-D-mannitol and [14C]-carboxyl-inulin across the blood-brain barrier and blood-spinal cord barrier in the rabbit. J Spinal Cord Med 1995; 18: 221-226.

35. Qureshi Al, Ali Z, Suri MF, Shuaib A, Baker G, Todd K, Guterman LR, Hopkins LN. Extracellular glutamate and other amino acids in experimental intracerebral hemorrhage: an in vivo microdialysis study. Crit Care Med 2003; 31: 1482-1489.

36. Rafałowska J, Sulejczak D, Chrapusta SJ, Gadamski R, Taraszewska A, Nakielski P, Kowalczyk T, Dziewulska D. Non-woven nanofiber mats - a new perspective for experimental studies of the central nervous system? Folia Neuropathol 2014; 52: 407-416.

37. Schellenberg AE, Buist R, Yong VW, Del Bigio MR, Peeling J. Magnetic resonance imaging of blood-spinal cord barrier disruption in mice with experimental autoimmune encephalomyelitis. Magn Reson Med 2007; 58: 298-305.

38. Schnell L, Fearn S, Klassen H, Schwab ME, Perry VH. Acute inflammatory responses to mechanical lesions in the CNS: differences between brain and spinal cord. Eur J Neurosci 1999; 11: 3648-3658.

39. Schousboe A, Sarup A, Bak LK, Waagepetersen HS, Larsson OM. Role of astrocytic transport processes in glutamatergic and GABAergic neurotransmission. Neurochem Int 2004; 45: 521527

40. Sharma HS. Pathophysiology of blood-spinal cord barrier in traumatic injury and repair. Curr Pharm Des 2005; 11: 13531389.

41. Sharp CD, Hines I, Houghton J, Warren A, Jackson TH 4th, Jawahar A, Nanda A, Elrod JW, Long A, Chi A, Minagar A, Alexander JS. Glutamate causes a loss in human cerebral endothelial barrier integrity through activation of NMDA receptor. Am J Physiol Heart Circ Physiol 2003; 285: H2592-H2598.

42. Sharp CD, Houghton J, Elrod JW, Warren A, Jackson TH 4th, Jawahar A, Nanda A, Minagar A, Alexander JS. N-methyl-D-aspartate receptor activation in human cerebral endothelium promotes intracellular oxidant stress. Am J Physiol Heart Circ Physiol 2005; 288: H1893-H1899.

43. Sheldon AL, Robinson MB. The role of glutamate transporters in neurodegenerative diseases and potential opportunities for intervention. Neurochem Int 2007; 51: 333-355.

44. Silver J, Miller JH. Regeneration beyond the glial scar. Nat Rev Neurosci 2004; 5: 146-156. 
45. Stys PK, Ransom BR, Waxman SG, Davis PK. Role of extracellular calcium in anoxic injury of mammalian central white matter. Proc Natl Acad Sci USA 1990; 87: 4212-4216.

46. Tsacopoulos M, Magistretti PJ. Metabolic coupling between glia and neurons. J Neurosci 1996; 16: 877-885.

47. Whetstone WD, Hsu JYC, Eisenberg M, Werb Z, Noble-Haeus slein LJ. Blood-spinal cord barrier after spinal cord injury: relation to revascularization and wound healing. J Neurosci Res 2003; 74: 227-239.

48. Yi JH, Hazell AS. Excitotoxic mechanisms and the role of astrocytic glutamate transporters in traumatic brain injury. Neurochem Int 2006; 48: 394-403. 